\title{
More accurate systolic blood pressure measurement is required for improved hypertension management: a perspective
}

This article was published in the following Dove Press journal:

Medical Devices: Evidence and Research

24 July 2017

Number of times this article has been viewed

\author{
Meir Nitzan' \\ Itzchak Slotki ${ }^{2}$ \\ Linda Shavit ${ }^{2}$ \\ 'Department of Applied Physics/ \\ Electro-Optics, Jerusalem College \\ of Technology, ${ }^{2}$ Department of \\ Nephrology, Shaare Zedek Medical \\ Center, Jerusalem, Israel
}

Correspondence: Meir Nitzan Department of Applied Physics/ElectroOptics, Jerusalem College of Technology, 2I Havaad Haleumi Street, Jerusalem, 91160 Israel

Tel +9722675 II 39

Fax +97226751045

Email nitzan@g.jct.ac.il
Abstract: The commonly used techniques for systolic blood pressure (SBP) and diastolic blood pressure (DBP) measurement are the auscultatory Korotkoff-based sphygmomanometry and oscillometry. The former technique is relatively accurate but is limited to a physician's office because its automatic variant is subject to noise artifacts. Consequently, the Korotkoff-based measurement overestimates the blood pressure in some patients due to white coat effect, and because it is a single measurement, it cannot properly represent the variable blood pressure. Automatic oscillometry can be used at home by the patient and is preferred even in clinics. However, the technique's accuracy is low and errors of 10-15 $\mathrm{mmHg}$ are common. Recently, we have developed an automatic technique for SBP measurement, based on an arm pressure cuff and a finger photoplethysmographic probe. The technique was found to be significantly more accurate than oscillometry, and comparable to the Korotkoff-based technique, the reference-standard for non-invasive blood pressure measurements. The measurement of SBP is a mainstay for the diagnosis and follow-up of hypertension, which is a major risk factor for several adverse events, mainly cardiovascular. Lowering blood pressure evidently reduces the risk, but excessive lowering can result in hypotension and consequently hypoperfusion to vital organs, since blood pressure is the driving force for blood flow. Erroneous measurement by $10 \mathrm{mmHg}$ can lead to a similar unintended reduction of SBP and may adversely affect patients treated to an SBP of 120-130 $\mathrm{mmHg}$. In particular, in elderly patients, unintended excessive reduction of blood pressure due to inaccurate SBP measurement can result in cerebral hypoperfusion and consequent cognitive decline. By using a more accurate technique for automatic SBP measurement (such as the photoplethysmographic-based technique), the optimal blood pressure target can be achieved with lower risk for hypotension and its adverse events.

Keywords: oscillometry, hypotension, cognitive impairment, photoplethysmography

\section{Perspectives}

The measurement of blood pressure (BP) is a mainstay for the diagnosis and follow-up of hypertension, which is a major risk factor for several adverse events, mainly cardiovascular. Lowering BP reduces the risk, but excessive lowering can result in deleterious side effects, such as hypoperfusion to vital organs, since BP is the driving force for blood flow. Accurate BP measurement is therefore essential for proper control of lowering BP. In the following perspective, we examine the accuracy of available techniques for non-invasive BP measurements and also assess the degree of accuracy required in BP measurement, in order to balance the benefit of BP reduction in hypertension against the risks of hypotension, when intensive treatment is implemented. We then present a 
novel application of a known technique for systolic BP (SBP) measurement, based on a pressure cuff wrapped around the arm and a photoplethysmographic probe on the finger distal to the cuff. In previous preliminary studies, it was found that the photoplethysmography (PPG)-based technique was significantly more accurate than the oscillometric technique and its accuracy was comparable to that of auscultatory Korotkoff sounds-based sphygmomanometry.

\section{Non-invasive BP measurement}

Each of the two common non-invasive techniques for BP measurement, the auscultatory sphygmomanometry and automatic oscillometry, has its own limitation with respect to accuracy. The auscultatory technique is considered as more accurate than the automatic oscillometric technique and is regarded as the gold reference for validation of automatic BP measurement devices, mainly based on oscillometry. However, the auscultatory technique cannot be performed automatically and is only suitable for the physician's office, where examinations may give falsely high readings due to white coat hypertension. Furthermore, office measurement only reflects SBP and diastolic BP (DBP) at a single time point, while both values change with time, an effect known as BP variability. The measurement at a single time point cannot reliably represent the actual variable $\mathrm{BP}$, and also cannot provide an assessment of the BP variability, an important clinical parameter by itself. ${ }^{1,2}$

Automatic BP measurement by oscillometry overcomes the two limitations of manual-auscultatory sphygmomanometry. In the oscillometric technique, air pressure pulses in the cuff during cuff deflation are analyzed and the mean BP, SBP and DBP are derived from the oscillometric waveform envelope of the cuff air pressure pulses, by means of various empirically derived algorithms. ${ }^{3,4}$ Oscillometric BP measurements can be done automatically at home, free of white coat effect, and since several measurements can be performed throughout the day, a more representative picture of $\mathrm{BP}$ than a single office BP measurement is obtained. Home BP seems to be more strongly associated with end-organ damage than office BP, and some studies (though not all) have suggested that home $\mathrm{BP}$ is a stronger predictor of future adverse cardiovascular events than office $\mathrm{BP}^{5-8}$

The disadvantage of oscillometry is that BP measurement by means of the technique is prone to significant errors. The indirect determination of SBP and DBP from the oscillometric waveform envelope depends on several physiologic parameters, such as pulse pressure, arterial compliance curve, soft tissue biomechanical properties, and heart rate. Altera- tions in these parameters change the oscillometric curve and, thereby, can result in significant errors in SBP and DBP readings. ${ }^{48-14}$ The multiplicity of algorithms for the evaluation of SBP and DBP from the air pressure oscillations ${ }^{4}$ also reflects and contributes to the uncertainty in the technique.

The low accuracy of oscillometric devices can also be inferred from the standards imposed by the British Hypertension Society (BHS) $)^{15}$ and the European Society of Hypertension (ESH). ${ }^{16}$ The protocols of both organizations are based on comparing the automated BP meter to the manual auscultatory technique. An automated BP measuring device can fulfill the BHS and ESH protocols, even if its readings deviate from those of the auscultatory technique by $10 \mathrm{mmHg}$ in $18 \%$ of the examinations (and also by $15 \mathrm{mmHg}$ in $5 \%$ of the examinations). Moreover, the auscultatory technique has its own deviation relative to the true gold standard, invasive measurement, so that the actual error in BP measurement by oscillometry is likely to be even greater than that permitted by the BHS and ESH.

In the following section, we propose that a potential error of $10 \mathrm{mmHg}$ in a significant number of examinations may adversely affect individual hypertensive patients undergoing treatment, mainly by leading to unintentional excessive lowering of BP.

\section{Accurate BP measurement in relation to clinical outcome}

Hypertension is generally defined as SBP/DBP values above $140 / 90 \mathrm{mmHg}$, and in most guidelines $140 / 90 \mathrm{mmHg}$ is the SBP/DBP target. Some guidelines recommend treating hypertensive persons aged 60 years or older to a BP goal of $150 / 90 \mathrm{mmHg},{ }^{17,18}$ but the high SBP target generated considerable controversy. ${ }^{19}$ For a patient treated to a BP target of 140-150/90, reduction of BP to a value of 130/80 seems to have only a minor effect, if any, on the blood perfusion to vital organs.

Taking this a step further, in the recent SPRINT multicenter intervention study ${ }^{20}$ on non-diabetic patients with increased cardiovascular risk, an intensive-treatment group (SBP target lower than $120 \mathrm{mmHg}$ ) was compared to a standard-treatment group (less than $140 \mathrm{mmHg}$ ). The intensive-treatment group had lower rates of fatal and non-fatal major cardiovascular events relative to the standard-treatment group. Similar results were obtained for a subgroup of the SPRINT study, which included ambulatory adults aged 75 years or older: intensive treatment resulted in significantly lower rates of fatal and non-fatal major cardiovascular events and death from any cause compared with standard treatment. ${ }^{21}$ 
On the other hand, patients in the intensive-treatment group had a higher rate of side effects related to hypotension, such as syncope and acute kidney injury. These adverse effects were considered to be of minor significance relative to the benefit of reduced risk of cardiovascular events. ${ }^{20,21}$ However, it is still possible that in intensively treated patients, further unintended reduction of BP by $10 \mathrm{mmHg}$ due to an error in measurement could have more serious clinical consequences.

The possibility of this hazard is supported by several studies which found higher risks of adverse cardiovascular events when BP was aggressively reduced. In several largescale trials on high cardiovascular risk patients, SBP values between 130 and $120 \mathrm{mmHg}$ were associated with an increase in the incidence of cardiovascular events, relative to SBP higher than $130 \mathrm{mmHg} .{ }^{22,23}$ Similar results were obtained in a recent study on Japanese older adults with isolated systolic hypertension treated with valsartan. ${ }^{24}$ Based on these studies, a higher risk of adverse events occurs when the measured SBP value is $130 \mathrm{mmHg}$, and if this value also includes a measurement error of $10 \mathrm{mmHg}$, then true SBP might be $120 \mathrm{mmHg}$.

The effect of erroneous excessive SBP reduction is of particular importance in relation to cognitive function in the elderly population. While it is accepted that hypertension in midlife is a risk factor for cognitive impairment and vascular dementia in late life, the effect of late-life BP on cognitive function is controversial. Some, but not all, studies found that hypertension or hypotension in late life is a risk factor for cognitive impairment and dementia. ${ }^{25-31}$ Hypotension is a potential risk factor for cerebral dysfunction and cognitive impairment, due to hypoperfusion, since arterial BP is the driving force for blood flow. A recent cohort study ${ }^{32}$ on patients with overt dementia and mild cognitive impairment found that patients with daytime SBP lower than $128 \mathrm{mmHg}$ showed a greater cognitive decline than those with higher SBP values. The association was significant only among patients treated with antihypertensive drugs. In another study, SBP of approximately $135 \mathrm{mmHg}$ was associated with optimal cognitive function in elderly African-Americans. ${ }^{33}$

The significance of the accuracy of SBP measurement for the assessment of the relationship between SBP and brain function can be inferred from a prospective cohort of elderly subjects during a 24-month follow-up, in which increased 24-hour ambulatory SBP readings, but not clinic SBP readings, were associated with increased white matter hyperintensity volume (measured by magnetic resonance imaging) over that same period. ${ }^{34}$ However, the use of ambulatory $\mathrm{BP}$ monitoring for routine detection and follow-up of BP is expensive and not convenient for practical routine use and, since it is based on the oscillometric method, it still has inherent inaccuracy. Although home BP measurement is a more practical method of follow-up, it is still oscillometry-based and, hence, subject to errors of the technique. Therefore, novel, more accurate techniques for automatic SBP measurement, in the home and clinic, are required for improving hypertension treatment.

\section{PPG-based SBP measurement}

We propose here an innovative approach to SBP measurement, which uses a pressure cuff wrapped around the arm and is based on direct detection of reopening of the artery under the cuff, when the air pressure decreases below the SBP value (similar to Korotkoff-based sphygmomanometry). The technique uses PPG, which measures changes of light transmission through tissue (e.g., the fingertip), due to cardiac-induced blood volume changes in the arteries. Figure 1 presents the PPG-based SBP measurement apparatus and Figures $2 \mathrm{~A}$ and $\mathrm{B}$ present the photoplethysmographic probe and signal. During systole, blood is ejected from the left ventricle and increases the arterial BP and consequently the volume of the arterial blood. Due to the systolic increase in tissue blood volume, the light transmission through the fingertip decreases during systole (Figure 2B). The SBP measurement technique is based on a pressure cuff around the arm and a photoplethysmographic probe on the fingertip distal to the cuff. When the cuff pressure increases to above SBP, the artery under the cuff collapses and the PPG signal disappears. The SBP value is taken as the value of the air pressure for which the PPG signal downstream to the cuff first reap-

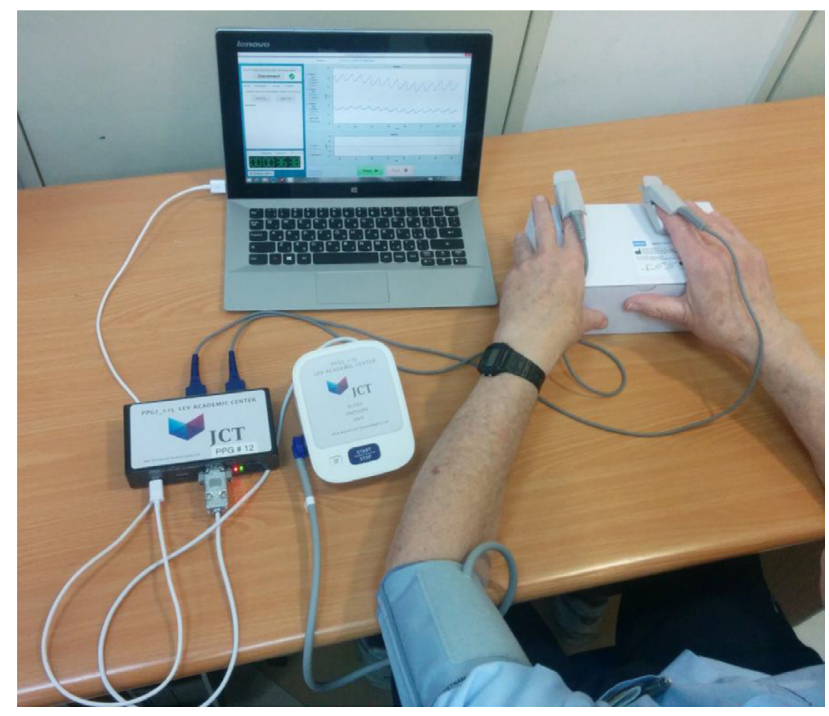

Figure I The PPG-based SBP measurement apparatus includes a pressure cuff wrapped around the arm and two photoplethysmographic probes on the fingers of the two hands.

Abbreviations: PPG, photoplethysmography; SBP, systolic blood pressure. 
A

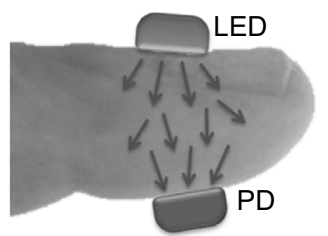

B

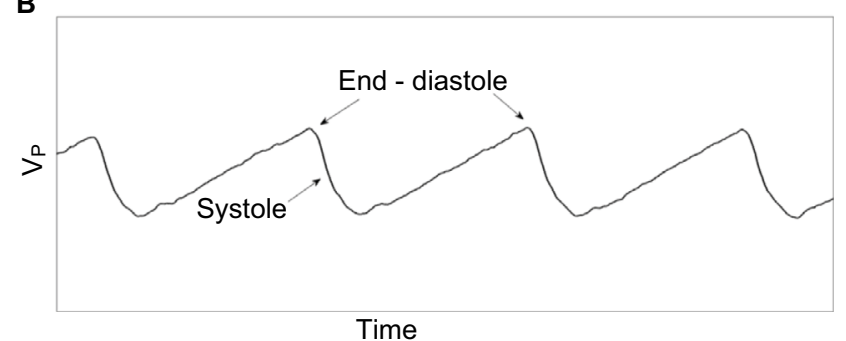

Figure 2 (A) The PPG probe. Light is transmitted from the light-source (LED) through the fingertip to the photodetector (PD). (B) Some pulses of the original PPG signal: the changes in transmitted light intensity as a function of time, due to cardiac activity. Maximal transmitted light intensity occurs at end diastole, when the tissue blood volume is at a minimum. $V_{p}$ is the voltage pulses measured by the photodetector PD.

pears during cuff deflation. ${ }^{35-37}$ In contrast to oscillometry, the technique enables SBP measurement with no need for a statistically based empirical formula. The PPG-based device is still investigational.

PPG is also the basis for the well-known pulse oximetry technique used in the determination of arterial oxygen saturation. The latter is derived from the PPG pulses in two wavelengths, using the different light absorption spectra in oxi- and deoxi-hemoglobin.

In general, the PPG pulses are small when the cuff pressure is slightly below the SBP value, and it is often difficult to differentiate between PPG pulses and noise fluctuations. In order to increase the reliability of the detection of these pulses, we added a photoplethysmographic probe in the free hand for confirmation that the detected fluctuations in the light transmission curve, measured in the finger distal to the cuff, are actual PPG pulses. Figure 3 presents the light transmission measurement from the two hands as a function of time during cuff deflation, at a time when cuff pressure is close to the SBP. For cuff pressure above SBP, no PPG pulses appear in the finger distal to the cuff, because of the collapse of the brachial artery under the cuff; when the cuff pressure decreases to below SBP value, the PPG pulses reappear. Note the delay in the arrival time of the PPG pulses in the finger distal to the cuff relative to that in the free hand, probably due to increase in compliance in the conduit arteries distal to the cuff. ${ }^{38}$

Detection of the reappearing PPG pulses can be done manually, by inspecting the light transmission curves during the deflation period, or automatically, using characteristic features of the PPG pulse. The automatic PPG technique was found to be significantly more accurate than oscillometry, using the auscultatory technique as the gold reference, as detailed in Table $1 .{ }^{39}$ In order to obtain the absolute accuracy of the PPG-based SBP measurement, a comparison with invasive measurement by arterial catheter - the gold standard - is required. However, the relative accuracy of the PPG-based SBP measurement technique and the auscultatory sphygmomanometry can be assessed without invasive BP measurements, using a non-invasive self-designed appraisal method (description follows). ${ }^{37}$

In the previously mentioned study, the PPG signals in the two hands were measured simultaneously by listening to Korotkoff sounds. The time point at which the first Korotkoff sound was heard, was digitally recorded, together with the two PPG curves (Figure 3). The study included 120

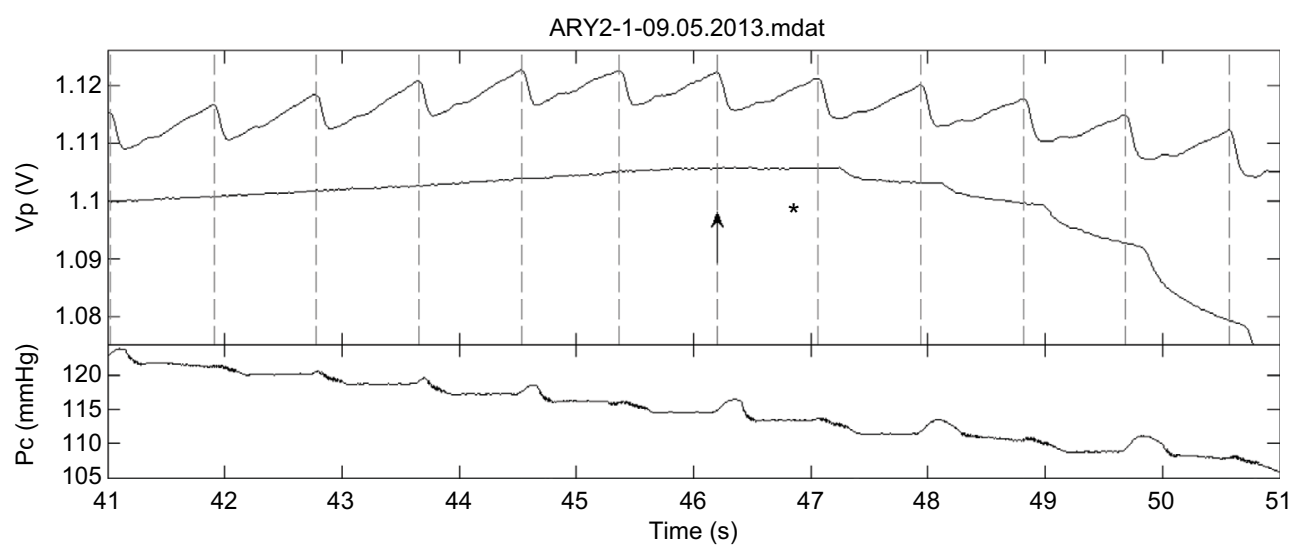

Figure 3 The PPG pulses $\left(V_{P}\right)$ in the two hands of one of the subjects during cuff deflation for cuff pressures $P_{C}$ in the neighborhood of the SBP value. The lower PPG curve is in the hand distal to the cuff and the upper one in the cuff-free hand. The vertical dotted lines indicate the start time of the systolic decrease in the cuff-free hand PPG pulses. The star indicates the time of the first detection of the Korotkoff sounds and the arrow indicates the start of the corresponding PPG pulse. In this examination, the first Korotkoff sounds were heard at nearly the same heart beat at which the first PPG pulse was detected. From Nitzan M, Adar Y, Hoffman E, et al. Comparison of systolic blood pressure values obtained by photoplethysmography and by Korotkoff sounds. Sensors (Basel). 2013;| 3(II): I 4797-I48I 2.37

Abbreviation: PPG, photoplethysmography; SBP, systolic blood pressure. 
Table I Results of mean difference, SD, and correlation coefficient of SBP measurement with the PPG-based technique and oscillometry as compared with those of auscultatory sphygmomanometry

\begin{tabular}{llll}
\hline & $\begin{array}{l}\text { Mean difference } \\
(\mathbf{m m H g})\end{array}$ & $\mathbf{S D}(\mathbf{m m H g})$ & $\begin{array}{l}\text { Correlation } \\
\text { coefficient }\end{array}$ \\
\hline PPG-based technique & 1.3 & 3.7 & 0.983 \\
Oscillometry & -4.9 & 7.3 & 0.934 \\
\hline
\end{tabular}

Notes: The mean difference and the SD for the PPG-based technique were smaller and the correlation coefficient was higher than those of oscillometry. Data from Nitzan M, Patron A, Glik Z, Weiss AT. Automatic noninvasive measurement of systolic blood pressure using photoplethysmography. Biomed Eng Online. 2009;8:28. ${ }^{39}$ Abbreviations: PPG, photoplethysmography; SBP, systolic blood pressure.

simultaneous examinations on 40 subjects. In the majority of examinations, the first detected PPG pulse appeared almost at the same time as the first Korotkoff sound was heard, indicating similar SBP values obtained by both techniques. In some examinations, however, differences in detection time between the two techniques were found. By finding the technique which first detects a pulse (either PPG or sound), the more accurate technique can be determined. When the first PPG pulses are observed during the cuff deflation, it is evident that the artery below the cuff has been reopened, and the SBP value is higher than the momentary cuff pressure. If Korotkoff sounds are not detected at that time, it is a failure of the auscultatory technique. Similarly, if Korotkoff sounds were heard in a given examination while no PPG pulse is detected, the Korotkoff technique was more accurate than the PPG technique in this examination. In that study, in 97 out of the 120 examinations, the difference between SBP values, measured by the two techniques, was less than 3 mmHg. In 13 examinations, SBP value measured by PPG was higher by more than $3 \mathrm{mmHg}$ than that measured by auscultation, while in ten examinations the PPG-based SBP value was lower by more than $3 \mathrm{mmHg}$ than that measured by auscultation. ${ }^{37}$

In summary, the PPG-based SBP measurement technique was found to be significantly more accurate than oscillometry (using Korotkoff-based technique as the reference standard) ${ }^{39}$ and was found to be as accurate as Korotkoff-based sphygmomanometry. ${ }^{37}$

The suggested PPG technique for SBP measurement is different from the volume clamp method, introduced by Jan Peňáz in 1973 for continuous non-invasive measurement of arterial BP, which is also based on PPG (Finapres and Finometer are representatives of this technique). The volume clamp technique is complicated relative to the other pressure cuff-based techniques: it uses a finger pressure cuff with a photoplethysmographic probe under it, and the cuff pressure is varied in time to counter the changing arterial BP during the cardiac cycle, keeping the blood volume constant. The method can provide BP pulse waveform as well as beat-to-beat SBP and DBP, but its accuracy regarding absolute values of SBP and DBP is limited. ${ }^{40,41}$ Our PPG-based technique for SBP measurement is based on a pressure cuff around the arm and a photoplethysmographic probe on a distal finger. It cannot measure BP continuously, but it can yield accurate absolute SBP measurement.

\section{Conclusion}

Since excessive lowering of BP can lead to adverse side effects, accurate measurement is essential for safe control of BP. However, each of the currently available non-invasive BP devices has its limitations in relation to accuracy. The auscultatory technique is only suitable for the physician's office, where examinations may give falsely high readings due to white coat hypertension and readings at only a single time point, which do not reflect BP variability. The automatic oscillometric technique can be used at home but is prone to significant errors, mainly due to the indirect determination of SBP and DBP from the oscillometric air pressure waves. An erroneously high reading of $10 \mathrm{mmHg}$ greater than the true SBP could lead to a correspondingly unintended reduction of SBP, so that a patient treated to a target of 120 or $130 \mathrm{mmHg}$ may have a true $\mathrm{BP}$ of $110-120 \mathrm{mmHg}$ with consequent adverse effects. A novel technique for SBP measurement, based on PPG, was found, in preliminary studies, to be apparently more accurate than oscillometry. The technique might enable more accurate automatic SBP measurements in the home and clinic, thereby allowing both optimal and safer control of SBP.

More accurate SBP measurement would provide the clinician with a better diagnostic tool for achieving the optimal SBP target and, hence, more effective management of hypertension. This is particularly important for the elderly, in whom accurate SBP measurement can minimize the short-term risks of hypotension and long-term risk of cognitive impairment due to excessive lowering of BP, while maximizing the cardiovascular benefits of treating hypertension.

\section{Disclosure}

Meir Nitzan has a US patent on a PPG-based systolic blood pressure meter and has also applied for another US patent. The authors report no other conflicts of interest in this work.

\section{References}

1. Stergiou GS, Ntineri A, Kollias A, Ohkubo T, Imai Y, Parati G. Blood pressure variability assessed by home measurements: a systematic review. Hypertens Res. 2014;37(6):565-572. 
2. McDonald C, Pearce MS, Kerr SR, Newton JL. Blood pressure variability and cognitive decline in older people: a 5-year longitudinal study. J Hypertens. 2017;35(1):140-147.

3. Ng KG, Small CF. Survey of automated noninvasive blood pressure monitors. J Clin Eng. 1994;19(6):452-475.

4. Forouzanfar M, Dajani HR, Groza VZ, Bolic M, Rajan S, Batkin I. Oscillometric blood pressure estimation: past, present, and future. IEEE Rev Biomed Eng. 2015;8:44-63.

5. Stergiou GS, Siontis KC, Ioannidis JP. Home blood pressure as a cardiovascular outcome predictor: it's time to take this method seriously. Hypertension. 2010;55(6):1301-1303.

6. Niiranen TJ, Thijs L, Asayama K, et al. The International Database of HOme blood pressure in relation to Cardiovascular Outcome (IDHOCO): moving from baseline characteristics to research perspectives. Hypertens Res. 2012;35(11):1072-1079.

7. Niiranen TJ, Hänninen MR, Johansson J, Reunanen A, Jula AM. Home-measured blood pressure is a stronger predictor of cardiovascular risk than office blood pressure: the Finn-Home study. Hypertension. 2010;55(6):1346-1351.

8. Ward AM, Takahashi O, Stevens R, Heneghan C. Home measurement of blood pressure and cardiovascular disease: systematic review and meta-analysis of prospective studies. J Hypertens. 2012;30(3):449-456.

9. Ursino M, Cristalli C. A mathematical study of some biomechanical factors affecting the oscillometric blood pressure measurement. IEEE Trans Biomed Eng. 1996;43(8):761-778.

10. van Popele NM, Bos WJ, de Beer NA, et al. Arterial stiffness as underlying mechanism of disagreement between an oscillometric blood pressure monitor and a sphygmomanometer. Hypertension. 2000;36(4):484-488.

11. van Montfrans GA. Oscillometric blood pressure measurement: progress and problems. Blood Press Monit. 2001;6(6):287-290.

12. Stergiou GS, Lourida P, Tzamouranis D, Baibas NM. Unreliable oscillometric blood pressure measurement: prevalence, repeatability and characteristics of the phenomenon. J Hum Hypertens. 2009;23(12):794-800.

13. Raamat R, Talts J, Jagomägi K, Kivastik J. Errors of oscillometric blood pressure measurement as predicted by simulation. Blood Press Monit. 2011;16(5):238-245.

14. Liu J, Hahn JO, Mukkamala R. Model-based error analysis of the oscillometric fixed-ratio blood pressure measurement method. Conf Proc IEEE Eng Med Biol Soc. 2012;2012:633-636.

15. O’Brien E, Petrie J, Littler WA, et al. The British Hypertension Society protocol for the evaluation of blood pressure measuring devices. $J$ Hypertens. 1993;11:S43-S62.

16. O'Brien E, Atkins N, Stergiou G, et al. European Society of Hypertension International Protocol revision 2010 for the validation of blood pressure measuring devices in adults. Blood Press Monit. 2010;15(1):23-38.

17. James PA, Oparil S, Carter BL, et al. 2014 evidence-based guideline for the management of high blood pressure in adults: report from the panel members appointed to the Eighth Joint National Committee (JNC 8). JAMA. 2014;311(5):507-520.

18. Qaseem A, Wilt TJ, Rich R, et al. Pharmacologic treatment of hypertension in adults aged 60 years or older to higher versus lower blood pressure targets: a clinical practice guideline from the American College of Physicians and the American Academy of Family Physicians. Ann Intern Med. 2017;166(6):430-437.

19. Reisin E, Harris RC, Rahman M. Commentary on the 2014 BP guidelines from the panel appointed to the Eighth Joint National Committee (JNC 8). J Am Soc Nephrol. 2014;25(11):2419-2424.

20. SPRINT Research Group; Wright JT Jr, Williamson JD, et al. A randomized trial of intensive versus standard blood-pressure control. N Engl J Med. 2015;373(22):2103-2116.
21. Williamson JD, Supiano MA, Applegate WB, et al. Intensive vs standard blood pressure control and cardiovascular disease outcomes in adults aged $\geq 75$ years: a randomized clinical trial. JAMA. 2016; 315(24):2673-2682.

22. Mancia G, Grassi G, Zanchetti A. Antihypertensive treatment and blood pressure in diabetic and nondiabetic patients: the lower, the better? Diabetes Care. 2011;34 Suppl 2:S304-S307.

23. Böhm M, Schumacher H, Teo KK, et al. Achieved blood pressure and cardiovascular outcomes in high-risk patients: results from ONTARGET and TRANSCEND trials. Lancet. 2017;389(10085):2226-2237.

24. Yano Y, Rakugi H, Bakris GL, et al. On-treatment blood pressure and cardiovascular outcomes in older adults with isolated systolic hypertension. Hypertension. 2017;69(2):220-227.

25. Qiu C, Winblad B, Fratiglioni L. The age-dependent relation of blood pressure to cognitive function and dementia. Lancet Neurol. 2005;4(8):487-499.

26. Tzourio C. Hypertension, cognitive decline, and dementia: an epidemiological perspective. Dialogues Clin Neurosci. 2007;9(1):61-70.

27. Moretti R, Torre P, Antonello RM, Manganaro D, Vilotti C, Pizzolato G. Risk factors for vascular dementia: hypotension as a key point. Vasc Health Risk Manag. 2008;4(2):395-402.

28. Kennelly SP, Lawlor BA, Kenny RA. Blood pressure and dementia - a comprehensive review. Ther Adv Neurol Disord. 2009;2(4):241-260.

29. Beeri MS, Ravona-Springer R, Silverman JM, Haroutunian V. The effects of cardiovascular risk factors on cognitive compromise. Dialogues Clin Neurosci. 2009;11(2):201-212.

30. Novak V, Hajjar I. The relationship between blood pressure and cognitive function. Nat Rev Cardiol. 2010;7(12):686-698.

31. Weiss J, Freeman M, Low A, et al. Benefits and harms of intensive blood pressure treatment in adults aged 60 years or older: a systematic review and meta-analysis. Ann Intern Med. 2017;166(6):419-429.

32. Mossello E, Pieraccioli M, Nesti N, et al. Effects of low blood pressure in cognitively impaired elderly patients treated with antihypertensive drugs. JAMA Intern Med. 2015;175(4):578-585.

33. Liu H, Gao S, Hall KS, et al. Optimal blood pressure for cognitive function: findings from an elderly African-American cohort study. $J$ Am Geriatr Soc. 2013;61(6):875-881.

34. White WB, Wolfson L, Wakefield DB, et al. Average daily blood pressure, not office blood pressure, is associated with progression of cerebrovascular disease and cognitive decline in older people. Circulation. 2011;124(21):2312-2319.

35. Talke PO. Measurement of systolic blood pressure using pulse oximetry during helicopter flight. Crit Care Med. 1991;19(7):934-937.

36. Langbaum M, Eyal FG. A practical and reliable method of measuring blood pressure in the neonate by pulse oximetry. J Pediatr. 1994;125(4):591-595.

37. Nitzan M, Adar Y, Hoffman E, et al. Comparison of systolic blood pressure values obtained by photoplethysmography and by Korotkoff sounds. Sensors (Basel). 2013;13(11):14797-14812.

38. Nitzan M, Rosenfeld C, Weiss AT, Grossman E, Patron A, Murray A. Effects of external pressure on arteries distal to the cuff during sphygmomanometry. IEEE Trans Biomed Eng. 2005;52(6):1120-1127.

39. Nitzan M, Patron A, Glik Z, Weiss AT. Automatic noninvasive measurement of systolic blood pressure using photoplethysmography. Biomed Eng Online. 2009;8:28.

40. Chin KY, Panerai RB. Comparative study of Finapres devices. Blood Press Monit. 2012;17(4):171-178.

41. Brittain JM, Busk TM, Møller S. Validation of non-invasive haemodynamic methods in patients with liver disease: the Finometer and the Task Force Monitor. Clin Physiol Funct Imaging. Epub 2017 Apr 12. 
Medical Devices: Evidence and Research is an international, peerreviewed, open access journal that focuses on the evidence, technology, research, and expert opinion supporting the use and application of medical devices in the diagnosis, monitoring, treatment and management of clinical conditions and physiological processes. The identification of novel devices and optimal use of existing devices which will lead to improved clinical outcomes and more effective patient management and safety is a key feature. The manuscript management system is completely online and includes a quick and fair peer-review system. Visit http://www. dovepress.com/testimonials.php to read real quotes from authors.

Submit your manuscript here: https://www.dovepress.com/medical-devices-evidence-and-research-journal 